\title{
SISTEMA PORFIRÍTICO YARUMALITO, ANTIOQUIA: PETROGRAFÍA Y CARACTERIZACIÓN DE LAS ALTERACIONES HIDROTERMALES
}

\author{
Marcela Barrera-Cortes ${ }^{1 *}$; José Carlos Frantz²; Juliana Charão-Marques²; Diana Sánchez-Celis ${ }^{1}$ \\ DOI: http://dx.doi.org/10.18273/revbol.v39n1-2017006 @ @ (i) \\ Forma de citar: Barrera-Cortes, M., Frantz, J.C., Charão-Marques, J., y Sánchez-Celis, D. 2017. Sistema \\ Porfirítico Yarumalito, Antioquia: Petrografía y caracterización de las alteraciones hidrotermales. Boletín de \\ Geología, 39(1): 127-136.
}

\section{RESUMEN}

En este trabajo se presenta la descripción petrográfica de secciones de núcleos (YAR-06, YAR-017 y YAR019) pertenecientes al Sistema Porfirítico Yarumalito, con el objeto de identificar la litología y alteraciones hidrotermales asociadas con mineralizaciones de oro en este sistema, propiedad de Colombian Mines Corporatión, este proyecto se localiza a 4,3Km al sur del municipio de Valparaíso, Antioquia. La descripción de estas secciones se realiza mediante el análisis macroscópico de núcleos de perforación, petrografía y microscopia electrónica (SEM); petrográficamente los cuerpos hipoabisales corresponden a andesitas y dacítas con alteraciones potásica, propilítica, cuarzo-sericita y sericítica; estás alteraciones tienen distribución en halos de vetillas o al interior de estas y menormente se presenta con una distribución penetrativa. En las secciones analizadas, el pulso inicial corresponde pórfidos de composición andesítica, con alteraciones potásica, cuarzo-sericitica y propilítica, este pulso es seguido por pórfidos dacíticos que presenta las mismas alteraciones pero con una intensidad mayor y finalmente estos cuerpos hipoabisales son cortados por pequeños diques dacíticos cuya alteración principal es sericitica. La mineralogía de los pórfidos aporta datos sobre las etapas evolutivas del sistema, así por ejemplo se observa como el pulso inicial es más básico y progresivamente va pasando a composiciones moderadamente más félsicas; de igual manera ocurre con las texturas de estos cuerpos, que sugieren niveles de profundidad relativamente diferentes para los pulsos identificados; por otra parte las texturas secundarias y las alteraciones presentes indican una prominente interacción con fluidos de origen magmático-hidrotermal.

Palabras clave: Petrografía, alteraciones hidrotermales, pórfidos, filosilicatos, Sistema porfirítico Yarumalito.

\section{YARUMALITO PORPHYRITIC SYSTEM, ANTIOQUIA: PETROGRAPHY AND CHARACTERIZATION OF HYDROTHERMAL ALTERATIONS ABSTRACT}

This paper describes petrographically sections of drill core (YAR-06, YAR-017 and YAR-019) belonging to the Yarumalite Porphyritic System, in order to identify the lithology and hydrothermal alterations associated with gold mineralization in this system, owned of Colombian Mines Corporation, this project is located 4,3 Km south of the municipality of Valparaiso, Antioquia. The description of these sections bodies is made by the macroscopic analysis of drilling cores, optical petrography and electron microscopy (SEM); petrographically the hypoabisal bodies correspond to andesites and dacites with potassic, propylitic, quartz-sericite and serictic alterations; these alterations have distribution in halos of veins or to the interior of these and less occurs with a distribution pervasive in the rock. In the analyzed sections, the initial pulse corresponds to andesitic porphyries, with potassic, quartzsericitic and propylitic alterations, this pulse is followed by dacitic porphyries that presents the same alterations but with a greater intensity and finally these hypoabisal bodies are cut by small dacitic dykes whose main alteration is sericitic. The mineralogy of the porphyries provides data on the evolutionary stages of the system, for example it is observed as the initial pulse is more basic and progressively goes to compositions moderately more felsic; similarly to the textures of these bodies, which suggest relatively different levels of depth for the identified pulses; on the other hand the secondary textures and hydrothermal alterations presents, suggest a prominent interaction with fluids of magmatic-hydrothermal origin.

Keywords: Petrography, hydrothermal alterations, porphyries, phyllosilicates, Porphyritic system Yarumalito.

\footnotetext{
${ }^{1}$ Programa de Pos graduación en Geociencias, Instituto de Geociencias, Universidad Federal do Rio Grande do Sul, Porto Alegre, RS, Brasil. (*) mbarreraco@gmail.com

${ }^{2}$ Laboratório de Geología Isotópica, Instituto de Geociencias, Universidad Federal do Rio Grande do Sul, Porto Alegre, RS, Brasil.jose.frantz@ufrgs.br, juliana.marques@ufrgs.br
} 


\section{INTRODUCCIÓN}

Los depósitos porfiríticos concentran cantidades considerables de metales como cobre, molibdeno y oro, mineralizaciones que aparecen tanto en las zonas de alteración hidrotermal como en intrusivos porfiríticos asociados (Sillitoe, 2008, 2010). El sistema porfitítico Yarumalito (SPY) es dominado por pórfidos de composición andesítica y dacítica, que localmente pueden presentar algunas variaciones texturales dando como resultado rocas con texturas más equigranulares. Thompson (2006) expone que la mineralización de oro en este sistema es controlada por la tendencia estructural W-NW y la clasificada en dos zonas: La zona mineralizada norte y la zona mineralizada sur.

Los datos de campo recolectados en el área de estudio por Colombian Mines Corporation (2010b) y Henrichs et al. (2014), junto con las descripciones de los núcleos y la petrografía óptica y electrónica (SEM) de secciones delgada pulidas, corroboran la presencia de rocas porfiríticas andesíticas y dacíticas mineralizadas.

Thompson (2006) indica que las vetas asociadas al sistema porfirítico Yarumalito fueron descubiertas por el señor Miguel Pérez en 1988, posterior al descubrimiento se inicia un periodo de minería artesanal que tomo auge entre 1990-1995; para el periodo de 1995-2002 Corona Goldfields S.A inició en conjunto con Miguel Pérez un proceso de exploración que culminó debido a la caída de los precios del oro y las condiciones de seguridad, finalmente en el año 2006 Colombian Mines Corporation inició actividades exploratorias.

Este trabajo presenta la descripción petrográfica tanto para la litología como para las alteraciones hidrotermales de secciones de tres núcleos de perforación del proyecto Yarumalito, estos análisis junto con datos de campo recolectados en el área de estudio por Colombian Mines Corporation (2010b) y Henrichs et al. (2014), permiten discriminar las zonas de alteración hidrotermal asociadas con los pulsos magmáticos presentes en la zona. Los resultados indican la presencia de tres pulsos $(P 1, P 2$ y $P 3$,) en los cuales se presenta oro, principalmente formando parte de la estructura de las piritas, en zonas de alteración potásica.

\section{CONTEXTO GEOLÓGICO LOCAL}

El SPY actualmente propiedad de Colombian Mines Corporation forma parte del distrito minero Marmato, este se localiza en el flanco oriental de la Cordillera Occidental entre los municipios de Caramanta y Valparaíso, departamento de Antioquia. Estos pórfidos atraviesan rocas volcano-sedimentarias y sedimentarias del Oligoceno Tardío y Mioceno, correspondientes a las Formaciones Amagá y Combia (FIGURA 1).

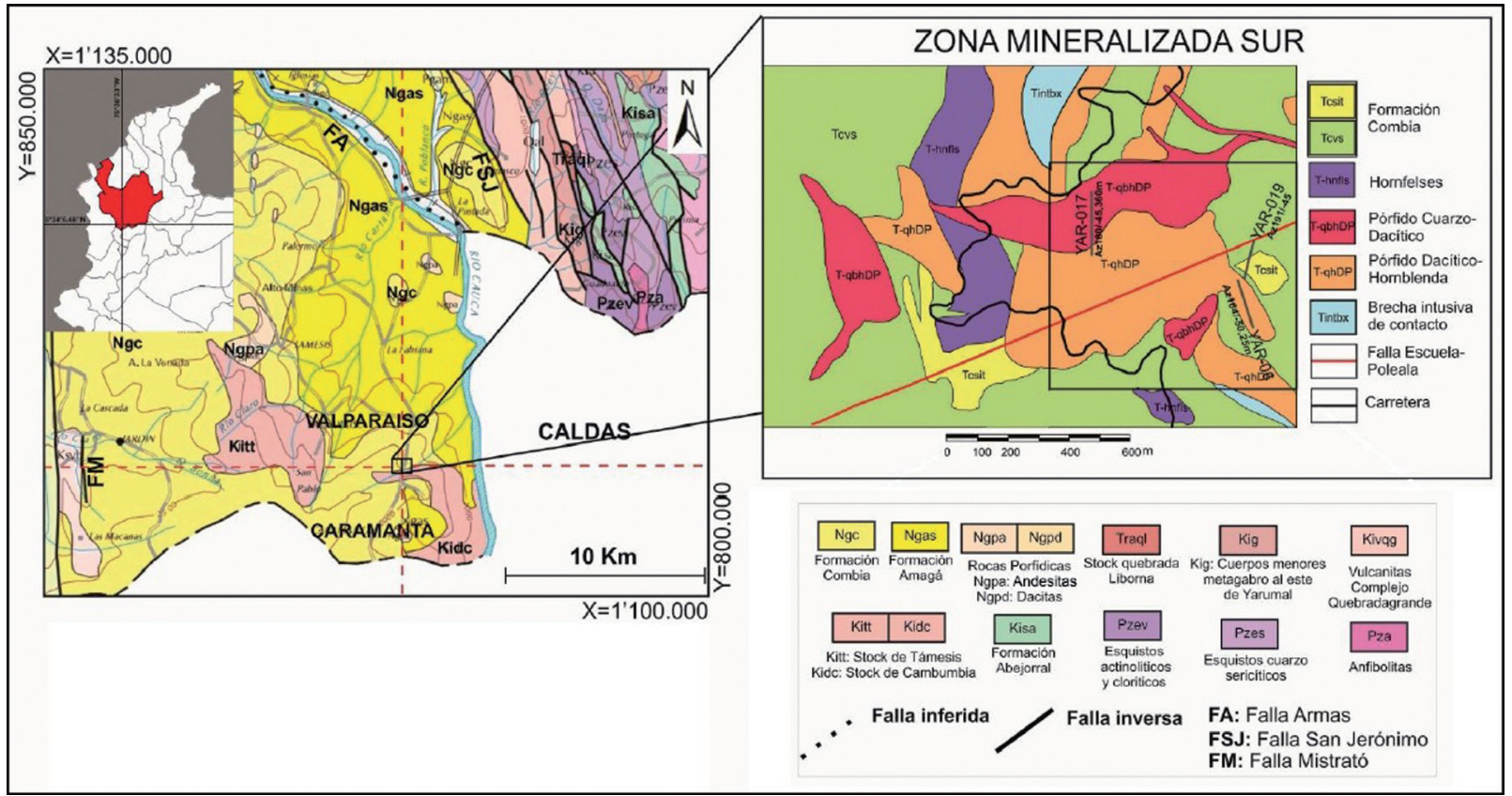

FIGURA 1. Localización del área de estudio. Modificado de González et al. (1999) y Colombian Mines Corporation (2010a). 
Las rocas porfiríticas del SPY en la zona de estudio, son cuerpos sub-volcanicos generalmente mineralizados de composición variable entre andesitas y dacitas, con alteraciones hidrotermales como: alteración potásica (Pot) con feldespato o biotita secundaria, alteración propilítica (Prop), alteración cuarzo sericitica (QzSer) y sericitización (Ser).

Chamois (2011) define la siguiente mineralización para estos cuerpos: pirita, calcopirita, pirrotita, esfalerita, molibdenita y oro libre con distribución en venas y vetillas de cuarzo, o diseminada.

\section{Formación Combia}

La Formación Combia corresponde a una sucesión volcano-sedimentaria, originada por varios pulsos magmáticos (López et al., 2006). El miembro volcánico de esta unidad consta esencialmente de flujos de lava, rocas piroclásicas y epiclásticas, el miembro sedimentario está constituido por niveles gruesos de conglomerados separados por finas capas de areniscas y arcillolitas (González, 2001).

La Formación Combia es generada en dos fases, la primera de vulcanismo efusivo con derrames basálticos, seguida por eventos de vulcanismo explosivo con emplazamiento de domos andesíticos que terminan siendo la fuente de los sedimentos del miembro clástico (González, 2001). Diversas dataciones realizadas en los cuerpos hipoabisales de esta formación sugieren edades del Mioceno Tardío, entre 12 y $6 \mathrm{Ma}(\mathrm{K} / \mathrm{Ar}$ en hornblenda: Restrepo et al., 1981; Trazas de fisión en circones: Ramírez et al., 2006; U/Pb en circones: Henrichs et al., 2014).

\section{Formación Amagá}

La Formación Amagá subyace discordantemente a la Formación Combia y es depositada en la cuenca interandina del Cauca (González, 2001). Esta unidad presenta mantos explotables de carbón y es conformada por sedimentos continentales fluviales y lacustres que son procedentes del Complejo Polimetamórfico de la Cordillera Central, el Granito de Amagá y el Complejo Quebradagrande (Calle y González, 1982; Guzmán-López, 2007). Van der Hammen (1958) propone una edad Oligoceno Medio a Mioceno Temprano para esta formación.

El depósito mineral Yarumalito se localiza sobre la depresión Cauca-Patía, que es interpretada en conjunto como una zona de sutura de edad Cretácico (González, 2001). Esta región es una zona tectónicamente activa, afectada principalmente por la Falla Cauca-Almaguer, una de las principales fallas que componen el Sistema de Fallas Romeral al noroccidente del país. Esta falla corresponde a la transición entre rocas oceánicas al occidente del sistema y rocas continentales al este (Paris et al, 2000). La Falla Cauca-Almaguer afecta principalmente a las Formaciones Amaga y Combia de edad Terciario, esta falla tiene movimiento lateral izquierdo con rumbo variable entre NW y NE (López et al, 2006).

Las mineralizaciones en SPY según Thompson (2006) están asociadas con una orientación W-NW. Esta dirección es concordante con el control estructural citado por varios autores (Molina, 1990; Alvarán, 2006; Santacruz, 2011) para algunas mineralizaciones en Frontino (Antioquía), Villamaría y Marmato (Caldas), localizadas en el cinturón metalógenetico del Cauca medio.

\section{METODOLOGÍA}

Para este estudio se contó con la colaboración de Colombian Mines Corporation que facilitó secciones de los núcleos de sondaje: YAR-06, YAR-017 y YAR-019, localizados sobre la zona mineralizada sur del SPY, en sectores donde el mapa MMI (Movile Metal Ion) para oro indica valores entre 0,5 y $0,1 \mathrm{ppb}$ (Colombian Mines Corporation, 2010a). Estas perforaciones tienen una profundidad máxima de $257 \mathrm{~m}, 339 \mathrm{~m}$, y $230 \mathrm{~m}$ respectivamente.

Los núcleos se describen macroscópica y microscópicamente, mediante el análisis de 30 secciones delgadas pulidas (TABLA 1); las abreviaciones para los minerales son tomadas de (Whitney and Evans, 2010). Para la petrografía se utilizó un microscopio Leica DM/LP y un equipo SEM (JSM 5800) del Instituto de Geociencias de la Universidad Federal do Rio Grande do Sul, las muestras fueron recubiertas (metalizadas) con carbono y se analizaron utilizando un voltaje de $15 \mathrm{kV}$ y una magnificación entre $5 \mu \mathrm{m}$ y $200 \mu \mathrm{m}$.

\section{RESULTADOS}

En las secciones analizadas se identifican tres cuerpos con textura porfirítica cuya relación matriz fenocristales se encuentra entre 1:9 hasta 2:3; la composición de estos pórfidos varía entre andesítica para el primer pulso $(P 1)$ y dacítica para los siguientes pulsos ( $P 2$ y $P 3)$, en estos pulsos es posible observar que las alteraciones del pórfido andesítico $(P 1)$ son obliteradas parcialmente por las alteraciones y la mineralización asociada con el pórfido dacítico $(P 2)$, el último pulso identificado corresponde a pórfidos dacíticos (P3) que cortan toda la secuencia. La composición modal y clasificación de estos cuerpos se muestran en la TABLA 2 y FIGURA 2. 
TABLA 1. Secciones delgadas pulidas (SDP) analizadas, indicando la profundidad de las mismas en los núcleos del SPY.

\begin{tabular}{c|cccccccc}
\hline & Prof.(m) & 69,38 & 72,17 & 111,4 & 125,44 & 133,87 & 172,74 & 181,59 \\
YAR-019 & SDP & L12 & L7 & L13 - L14-L15 & L22 & L31 & L16-L17- L18 & L19-L20 \\
& Prof.(m) & 186,28 & 186,72 & 203,4 & 228,15 & --- & -- & -- \\
& SDP & L8 & L9 & L28 & L10 & --- & --- & -- \\
YAR-017 & Prof.(m) & 83,63 & 128,47 & 196,74 & 233,86 & 278,35 & --- & -- \\
& SDP & L6 & L21 & L3- L4 & L11 & L27 & --- & -- \\
YAR.-06 & Prof.(m) & 101,52 & 141,51 & 233,29 & 166,63 & 179 & 201,31 & 207,7 \\
& SDP & L1 & L24- L25 & L5 & L30 & L2 & L29 & L26 \\
\hline
\end{tabular}

TABLA 2. Porcentaje modal de los minerales de las rocas del SPY (* indica los minerales de ganga asociados con las alteraciones hidrotermales).

\begin{tabular}{|c|c|c|c|c|c|c|c|c|c|c|c|}
\hline \multirow{3}{*}{$\begin{array}{l}\text { Unidad } \\
\text { Mineral }\end{array}$} & \multicolumn{5}{|c|}{ P1 } & \multicolumn{5}{|c|}{ P2 } & \multirow{2}{*}{$\frac{\text { P3 }}{\text { YAR19 }}$} \\
\hline & YAR06 & YAR17 & YAR17 & YAR06 & YAR06 & YAR19 & YAR19 & YAR19 & YAR19 & YAR19 & \\
\hline & L2 & L3 & L4 & L5 & L1 & L10 & L19 & L14 & L12 & L8 & L7 \\
\hline $\mathrm{Pl}$ & 40 & 37 & 46 & 40 & 50 & 40 & 35 & 42 & 29 & 40 & 55 \\
\hline Qz & 10 & 8 & 8 & 6 & 10 & 15 & 12 & 14 & 13 & 15 & 20 \\
\hline Or* & 1 & 1 & 2 & 1 & 1 & 0 & 0 & 0 & 1 & 0 & 10 \\
\hline Amp & 4 & 6 & 3 & 5 & 4 & 2 & 0 & 0 & 0 & 0 & 0 \\
\hline $\mathrm{Bt}$ & 3 & 3 & 3 & 3 & 0 & 1 & 3 & 0 & 1 & 2 & 0 \\
\hline Zrn & 1 & 1 & 1 & 1 & 1 & 0 & 1 & 0 & 1 & 0 & 0 \\
\hline Ap & 0 & 1 & 1 & 1 & 1 & 1 & 0 & 0 & 0 & 1 & 0 \\
\hline $\mathrm{Mt}$ & 2 & 2 & 2 & 3 & 3 & 2 & 2 & 0 & 1 & 2 & 0 \\
\hline Py & 2 & 3 & 3 & 5 & 2 & 1 & 2 & 2 & 0 & 2 & 0 \\
\hline Ccp & 0 & 2 & 2 & 2 & 2 & 0 & 1 & 1 & 1 & 1 & 0 \\
\hline $\mathrm{Cb}^{*}$ & 4 & 5 & 3 & 2 & 3 & 0 & 2 & 2 & 1 & 0 & 0 \\
\hline Ep* & 0 & 2 & 2 & 0 & 1 & 0 & 1 & 1 & 0 & 1 & 0 \\
\hline $\mathrm{Ch}{ }^{*}$ & 3 & 2 & 2 & 1 & 2 & 1 & 0 & 2 & 0 & 1 & 0 \\
\hline Mica blanca* & 0 & 2 & 2 & 0 & 0 & 0 & 1 & 1 & 0 & 0 & 5 \\
\hline Act* & 0 & 0 & 0 & 0 & 0 & 2 & 0 & 0 & 0 & 0 & 0 \\
\hline Fenoxtales $(\%)$ & 70 & 75 & 80 & 70 & 80 & 65 & 60 & 65 & 50 & 65 & 90 \\
\hline Matriz & 30 & 25 & 20 & 30 & 20 & 35 & 40 & 35 & 50 & 35 & 10 \\
\hline Total & 100 & 100 & 100 & 100 & 100 & 100 & 100 & 100 & 100 & 100 & 100 \\
\hline
\end{tabular}

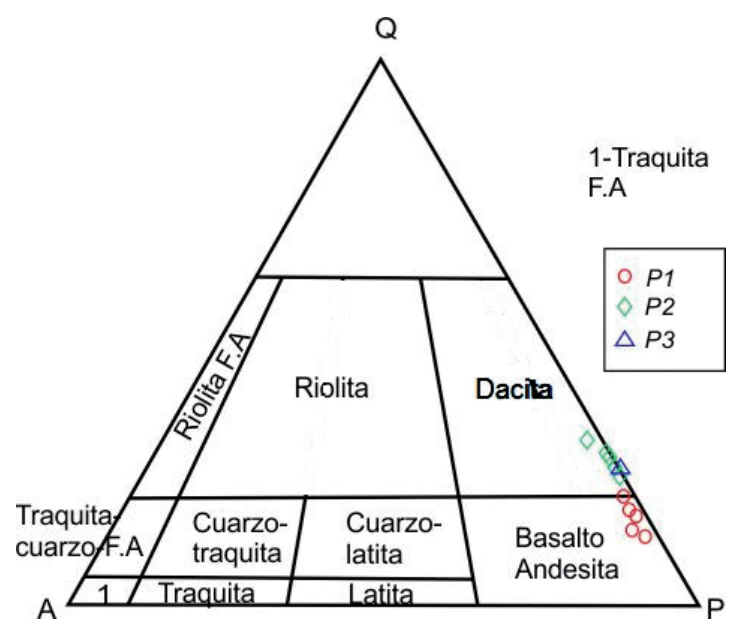

FIGURA 2. Diagrama de clasificación de rocas volcánicas de Streckeisen (1978) para los pórfidos analizados del SPY. Clasificación de las muestras con base en un conteo de 200 puntos.

\section{Petrografía}

\section{Pórfido andesítico (P1)}

La matriz de esta roca alcanza un $25 \%$, es principalmente microcristalina y está constituida por plagioclasas anhedrales a subhedrales con sericitización incipiente; biotitas anhedrales y algunas hornblendas moderada a fuertemente alteradas a biotita secundaria; esta roca tiene estructura masiva, inequigranular de tamaño de grano medio a grueso y textura general hipidiomórfica, con presencia escasa de texturas de exsolución (antipertita) en plagioclasas.

La mineralogía es plagioclasa, cuarzo, anfíbol y biotita, siendo este último el mineral máfico de mayor predominio tanto en la matriz como en los fenocristales. Como minerales accesorios se encuentran circones y apatitos. Como minerales de alteración aparece biotita 
secundaria, clorita y mica blanca; las dos primeras asociadas al reequilibrio de hornblendas y biotitas magmáticas o como minerales neoformados producto de la alteración hidrotermal y la mica blanca como producto de la alteración hidrotermal de plagioclasas.

Las plagioclasas varían de oligoclasas hasta andesinas (según el método de Michel-Levy), corresponde a 60\% en cristales euhedrales, prismáticos con tamaño entre $3 \mathrm{~mm}$ y $6 \mathrm{~mm}$ con zonación compleja, frecuentemente inversa y con macla polisintética. La biotita representa el $15 \%$ en cristales tabulares subhedrales hasta anhedrales con tonalidades café-verdosas, con tamaños de $0,5 \mathrm{~mm}$ a $1 \mathrm{~mm}$, se pueden encontrar cristales moderada y levemente cloritizados. Los anfíboles corresponden a $10 \%$ en cristales anhedrales con tamaño hasta de $1 \mathrm{~mm}$ que varían de verde claro a verde oscuro, frecuentemente con bordes reabsorbidos y alterados a biotita y menormente a clorita. El cuarzo representa $10 \%$ de los fenocristales, generalmente anhedrales con tamaño inferior a $0,5 \mathrm{~mm}$, extinción ondulante y bordes reabsorbidos (FIGURA 3A, 3B).

Los minerales accesorios representan el $2 \%$ y corresponde a cristales anhedrales de circón cuya coloración varía de incoloro a castaño claro y apatito con tamaños que no sobrepasan $0,05 \mathrm{~mm}$; el $3 \%$ restante corresponde a Py, Ccp y Mgt diseminadas y menormente en vetillas.

\section{Pórfido dacítico (P2)}

Presentan una estructura masiva, holocristalina, hipidiomorfa, con tamaño de grano medio a grueso, tiene un $40 \%$ de matriz cuya textura es criptocristalina y está conformada por plagioclasas subhedrales a anhedrales, cuarzo anhedral con leve extinción ondulante, biotita subhedral levemente cloritizada, anfíboles subhedrales menormente biotitizados y cloritizados y minerales opacos (Py) euhedrales a subhedrales. Como minerales accesorios aparecen circón y apatito y como minerales de alteración son frecuentes, mica blanca asociada a plagioclasas y biotita-clorita asociadas a minerales máficos.

Los fenocristales corresponden a $50 \%$ plagioclasa de tipo oligoclasa (Michel-Levy), con formas subhedrales a euhedrales, prismáticos con tamaño entre $1 \mathrm{~mm}$ a $3 \mathrm{~mm}$, con zonación compleja, generalmente normal y maclas polisintética y de Carlsbad; es frecuente encontrar cristales parcial a totalmente sericitizados. El cuarzo corresponde a $20 \%$ de fenocristales subhedrales con tamaños de $0,5 \mathrm{~mm}$ a $1 \mathrm{~mm}$, extinción ondulante y bahías de corrosión. El 15\% de los cristales son de biotita verdosa tabular, subhedral con tamaño de $1 \mathrm{~mm}$ a $3 \mathrm{~mm}$ y frecuentes reemplazamientos por clorita. El anfibol representa $10 \%$ de cristales anhedrales a subhedrales fuertemente fracturados y con tamaño de $1 \mathrm{~mm}$, es común encontrar cristales parcialmente biotitizados y cloritizados (FIGURA 3C, 3D).

Los minerales accesorios son apatito y circón incoloro a castaño claro que se encuentra ocasionalmente como inclusiones en biotitas y anfiboles, estos corresponden a un $1 \%$, mientras el $4 \%$ restante corresponde a Py, Ccp y Mgt.

\section{Pórfido dacítico (P3)}

Aparece muy localmente intruyendo el pulso $P 2$; son rocas holocristalinas, xenomórficas con estructura masiva y tamaño de grano fino a medio, la matriz es microcristalina y relativamente escasa $\leq 10 \%$. La mineralogía tanto en la matriz como en los fenocristales corresponde a plagioclasa, cuarzo y feldespato potásico. La alteración presente es una sericitización intensa de plagioclasas y como halos de alteración en los contactos con $P 2$.

El cuarzo corresponde a $20 \%$ de fenocristales anhedrales con tamaño de $0,1 \mathrm{~mm}$ a $0,5 \mathrm{~mm}$, con extinción fuertemente ondulante. El feldespato potásico representa $10 \%$ de los fenocristales con formas subhedrales y frecuente macla de Carlsbad y tamaños que alcanzan $1 \mathrm{~mm}$. El 55\% restante corresponde a plagioclasa subhedral, con macla polisintética, cuyos cristales están orientados paralelamente a la dirección de la intrusión. La alteración hidrotermal se presenta como aglomerados de mica blanca con cristales de $1 \mathrm{~mm}$ o menores y como halos de alteración alrededor del pórfido (FIGURA 3E, 3F).

\section{Alteraciones hidrotermales y mineralización asociada}

En el pórfido $P 1$ se reconocen: alteración potásica (Pot) con la paragénesis BtII+Fsp II (remanentes) $+\mathrm{Chl} \mathrm{I}$; alteración propilítica (Prop) cuya paragénesis es Chl $\mathrm{II}+\mathrm{Cb}+$ Ep y alteración cuarzo-sericitica (QzSer) en la cual se presenta Qz+mica blanca+Py. En el pórfido P2 se reconocen claramente: alteración Pot., alteración Prop. y alteración QzSer. (FIGURA 4), mientras en el pórfido $P 3$ solo se reconoce sericitización (Ser).

La alteración potásica de los pórfidos $P 1$ y $P 2$ es caracterizada por la presencia conspicua de Bt secundaria subhedral a anhedral, diseminada, con tamaños entre $<5 \mu \mathrm{m}$ hasta $20 \mu \mathrm{m}$, los cristales de mayor tamaño tienen reemplazamiento puntual por clorita y óxidos de hierro, comúnmente esta mica forma glomeropórfidos y cumulopórfidos junto con Mgt, Py y menormente Ccp; en $P 1$ se encuentras Bt secundarias formadas por reequilibrio de las hornblendas y aparecen remanentes de Fsp secundario. 

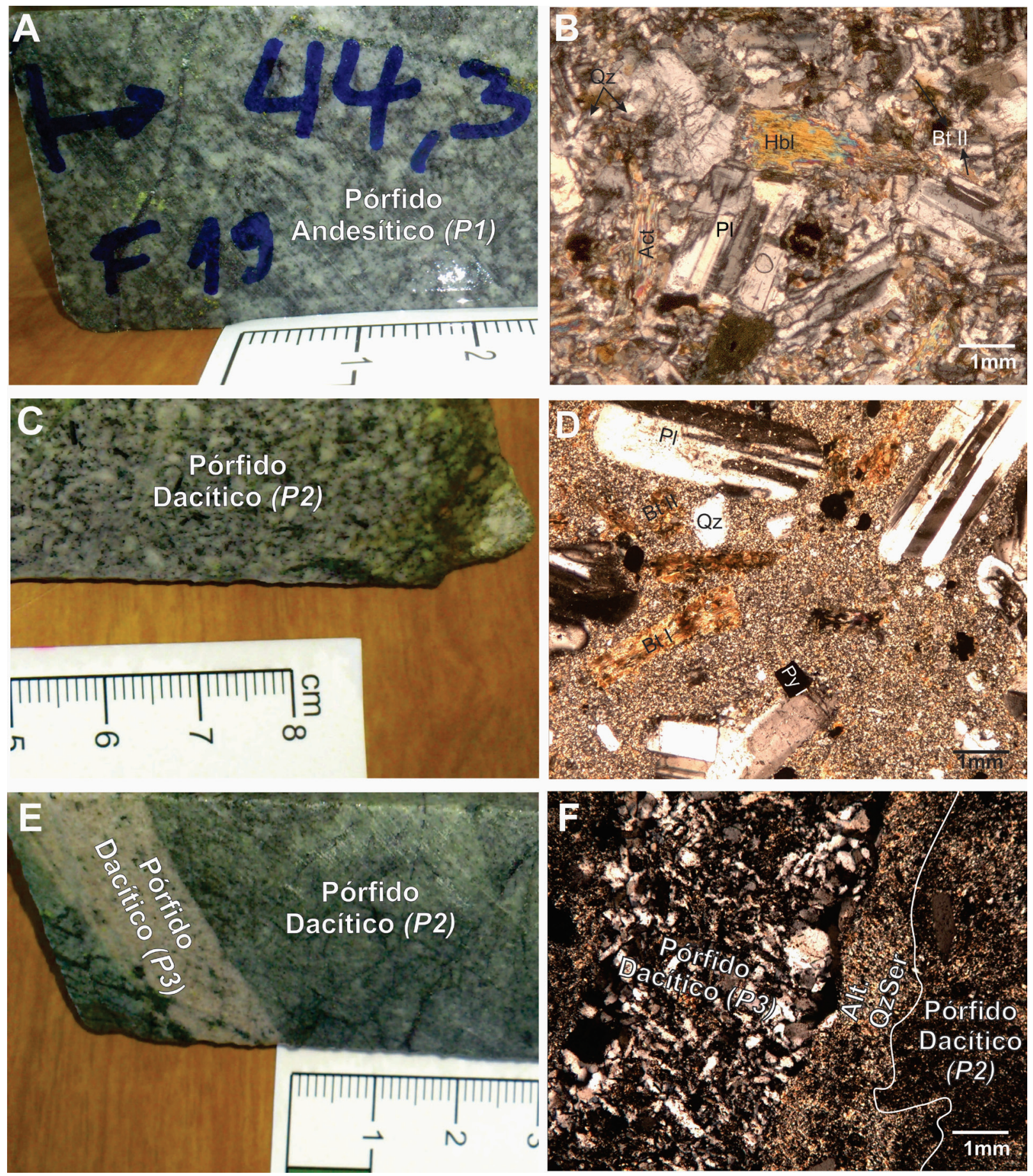

FIGURA 3. Secciones macroscópicas y microscópicas de los pórfidos andesíticos y dacíticos analizados. A y B. Pórfido andesítico (P1). C y D. Pórfido dacítico (P2). E y F. Pórfido dacítico (P3). 


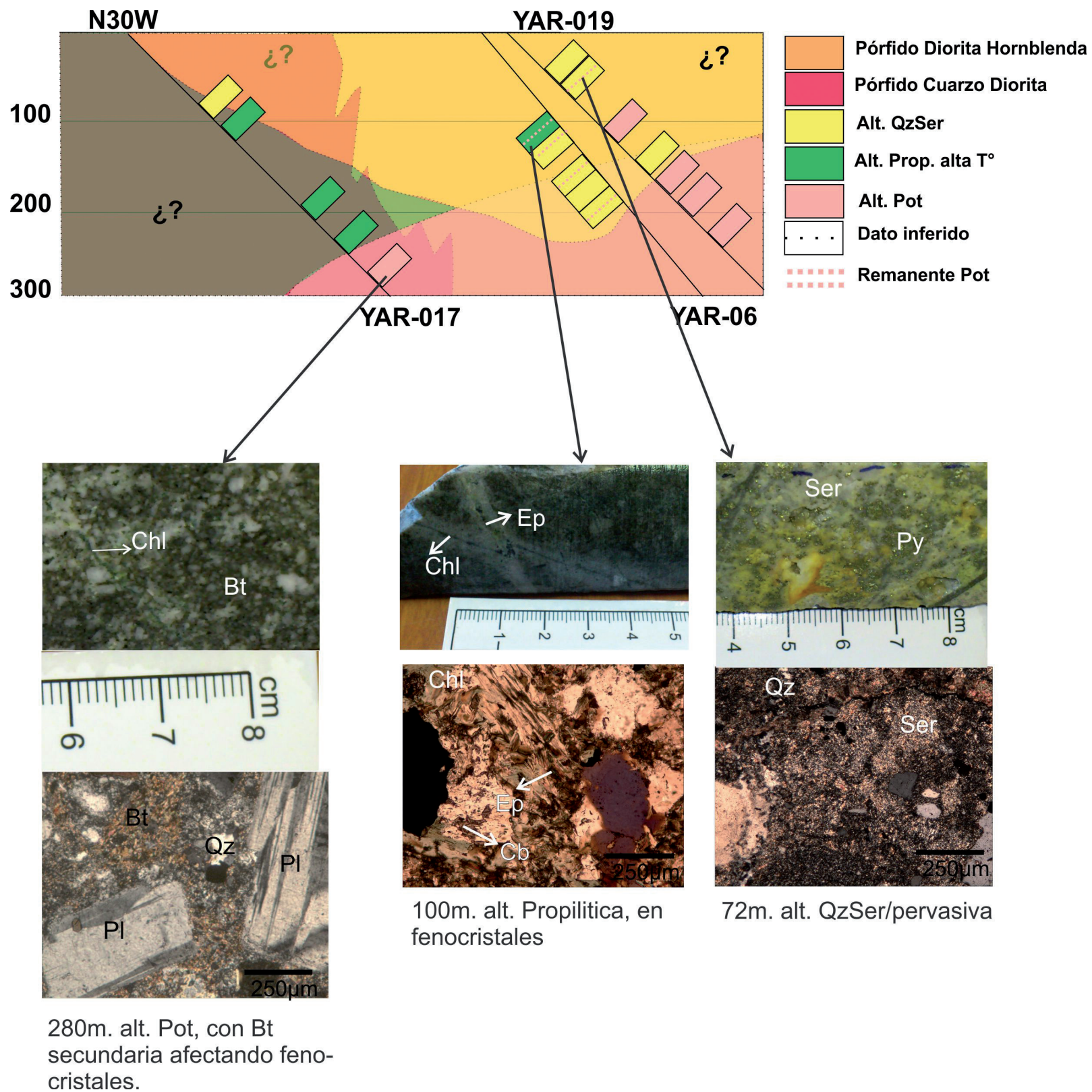

FIGURA 4. Esquema de distribución de alteraciones hidrotermales en la zona del SPY. Los polígonos punteados son interpretativos. Los datos de litología corresponden a los presentados para la zona por Colombian Mines.

Una asociación mineral frecuentemente encontrada en la alteración potásica del $P 2$ es: clorita y actinolita (posibles relictos de una alteración propilítica de mayor temperatura), que aparecen al interior de vetillas y como parte de halos de alteración. Esta alteración se asocia con vetillas $\mathrm{Qz} \pm \mathrm{Cb} \pm \mathrm{Mgt} \pm \mathrm{Py} \pm \mathrm{Ccp}$.

La clorita aparece en los pulsos $P 1$ y $P 2$ en las siguientes asociaciones minerales:
1. Clorita 1 (Chl I): Asociación de alta temperatura, aparece junto con $\mathrm{Bt}$, carbonato, actinolita (en P2) y epidota, pirita, magnetita y calcopirita (en P1). La clorita exhibe un hábito fibroso con tamaños de $50 \mu \mathrm{m}$ dentro de vetillas junto con cuarzo o en los halos de alteración de las mismas, es menos frecuente encontrar la clorita como cristales diseminados.

2. Clorita 2 (Chl II): Alteración propilítica, notoria especialmente en $P 1$ donde presenta una textura de 
brecha hidrotermal. Se encuentra en paragénesis con epidota, carbonato, magnetita (con inclusiones de Ccp y Py). Carbonato y epidota generalmente son cristales subhedrales con tamaño mayor a $50 \mu \mathrm{m}$, mientras la clorita se encuentra como agregados cristalinos anhedrales con pleocroísmo que varía de verde pálido a verde oscuro.

3. Clorita 3 (Chl III): Aparece en P2, sugiriendo bajas temperaturas de formación, debido a su asociación con mica blanca. La clorita forma vetillas o se encuentra como agregados cristalinos con hábito fibroso con tamaños entre $20 \mu \mathrm{m}$ y $30 \mu \mathrm{m}$, con tonos verdosos pálidos y pleocroísmo bajo.

La alteración QzSer, se identifica en el pórfido $P 1$ con la paragénesis mica blanca \pm clorita $(\mathrm{Chl}$ III $) \pm$ pirita \pm calcopirita. Esta mica blanca es incolora a verde claro y se encuentra como agregados cristalinos finos con tamaño menor o igual a $20 \mu \mathrm{m}$, aparece diseminada o como halos de alteración asociados con vetillas tardías, es frecuente encontrarla de forma pervasiva sobre alúminosilicatos.

En el pórfido dacítico $P 3$ la sericitización es representada por mica blanca diseminada como agregados fibrosos radiales con tamaño menor a $20 \mu \mathrm{m}$ y como halos de alteración en los contactos intrusivos con $P 2$.

A las mineralizaciones de Py, Mgt y Ccp asociadas con las alteraciones hidrotermales (Pot, Propi y QzSer) de $P 1$ y $P 2$, (FIGURA 5), se les realizan análisis EDS (TABLA 3), con el fin de tener una idea de posibles anomalías elementares en su estructura, cabe destacar que este tipo de análisis es cualitativo.
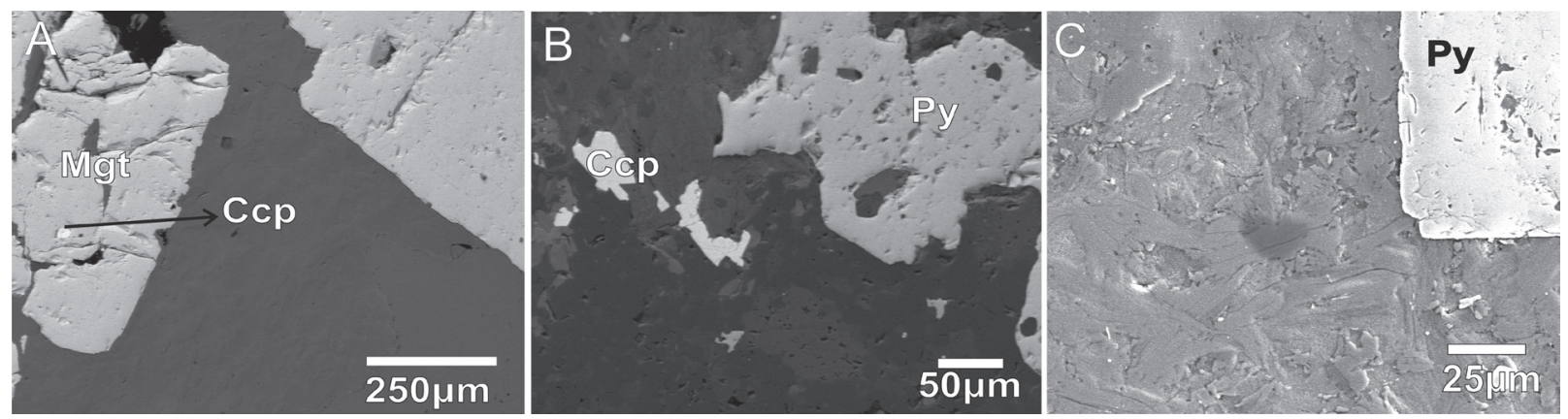

FIGURA 5. Imágenes BSE y SEI de mineralizaciones asociadas con las alteraciones hidrotermales del pórfido P2. A. alteración Prop. B. alteración Pot. C. alteración QzSer.

TABLA 3. Análisis EDS para Py Cep y Mgt de los pulsos porfiríticos $P 1, P 2$ y $P 3$ del SPY.

\begin{tabular}{|c|c|c|c|c|c|c|c|c|c|}
\hline \multirow{2}{*}{ Pórfido } & \multirow{2}{*}{ Núcleo } & \multirow{2}{*}{ SDP } & \multirow{2}{*}{ Mineral } & \multicolumn{6}{|c|}{ Elementos } \\
\hline & & & & $\mathrm{S}$ & $\mathrm{Fe}$ & $\mathrm{Au}$ & $\mathrm{Cu}$ & Co & $\mathrm{Ag}$ \\
\hline \multirow{8}{*}{$P 1$} & \multirow{3}{*}{ YAR-06 } & 2 & $\mathrm{Py}$ & $\mathrm{x}$ & $\mathrm{x}$ & $\mathrm{x}$ & & \multirow{3}{*}{$\mathrm{x}$} & \\
\hline & & 29 & Py & $\mathrm{x}$ & $\mathrm{x}$ & $\mathrm{x}$ & & & $\mathrm{x}$ \\
\hline & & 29 & Ccp & $\mathrm{x}$ & $\mathrm{x}$ & $\mathrm{x}$ & $\mathrm{x}$ & & $\mathrm{x}$ \\
\hline & \multirow{5}{*}{ YAR-017 } & 4 & Ccp & $\mathrm{x}$ & $\mathrm{x}$ & $\mathrm{x}$ & $\mathrm{x}$ & \multirow[b]{5}{*}{$\mathrm{x}$} & \\
\hline & & 4 & Mgt & & $\mathrm{x}$ & & & & \\
\hline & & 11 & Mgt & & $\mathrm{x}$ & $\mathrm{x}$ & & & \\
\hline & & 11 & Ccp & $\mathrm{x}$ & $\mathrm{x}$ & & $\mathrm{x}$ & & \\
\hline & & 21 & Py & $\mathrm{x}$ & $\mathrm{x}$ & & & & \\
\hline \multirow{3}{*}{$P 2$} & \multirow{3}{*}{ YAR-019 } & 8 & Mgt & & $\mathrm{x}$ & & & \multirow{3}{*}{$\mathrm{x}$} & \\
\hline & & 8 & Py & $\mathrm{x}$ & $\mathrm{x}$ & & & & \\
\hline & & 8 & Ccp & $\mathrm{x}$ & $\mathrm{x}$ & $\mathrm{x}$ & $\mathrm{x}$ & & \\
\hline
\end{tabular}

La magnetita (con frecuentes inclusiones de Ccp) aparece como cristales generalmente anhedrales con contactos ameboidales, cuyo tamaño no supera los $350 \mu \mathrm{m}$; está asociada con las alteraciones Pot y Prop., siendo más abundante en esta última.
La pirita en las paragénesis de alta temperatura se presenta como cristales anhedrales, con texturas ameboidales, formando vetillas incipientes, generalmente está acompañada de Ccp (inclusiones) y presenta algunas trazas de Ag y menos comúnmente Co, siendo según 
Ramdohr (1980 en Alvarán, 2006) la presencia de este último un rasgo poco frecuente en la estructura de las piritas. En las paragénesis de menor temperatura los cristales de Py son subhedrales a euhedrales con tamaños que alcanzan $250 \mu \mathrm{m}$ a $300 \mu \mathrm{m}$.

Calcopirita, aparece formando pequeñas inclusiones dentro de magnetita y pirita o diseminada en la roca, en ambos casos se presenta como cristales anhedrales con tamaño menor a $50 \mu \mathrm{m}$, con trazas de $\mathrm{Ag}$ y Au.

\section{ANÁLISIS DE RESULTADOS}

Los pórfidos $P 1$ y $P 3$ tienen una textura similar (con fenocristales poco desarrollados, dando una apariencia principalmente equigranular), sugiriendo que la cristalización de estos dos cuerpos se dió bajo condiciones similares, mientras el pórfido $P 2$ presenta una relación matriz fenocristales cercana a 2:3, con microfenocristales de mayor tamaño a los de los pórfidos anteriormente descritos, indicando que la cristalización ocurrió bajo condiciones diferentes a las observadas en los dos pórfidos anteriormente mencionados ( $P 1$ y $P 3$ ).

Las texturas de corrosión son frecuentes en fenocristales del pórfido $P 1$, indicando un fuerte desequilibrio entre el fundido que formó los fenocristales y el fundido remanente que forma la matriz; sin embargo, LópezRendón y Bedoya (1989 en Vargas, 2005) sugieren la posibilidad de que este tipo de corrosión pueda ser generada por la interacción con fluidos hidrotermales posteriores.

Los fluidos hidrotermales afectan principalmente los pórfidos $P 1$ y $P 2$, siendo $P 2$ el relacionado con la fase mineralizante; en estos pórfidos las mineralizaciones se encuentran asociadas a las fases de alteración tardimagmática de mayor temperatura, como las alteraciones Pot y Prop., a las cuales están asociadas cantidades considerables de Py, Mgt y Ccp con algunas trazas menores de $\mathrm{Au}$.

La aparición de minerales secundarios asociados a las zonas de alteración hidrotermal, como biotita, clorita y moscovita, sumado a la asociación de estos Mgt, Py y menormente Ccp, podría sugerir un aumento en la concentración de elementos como Fe y Mg durante los procesos hidrotermales.

\section{CONCLUSIONES}

Las variaciones texturales de los pórfidos $P 1$ y $P 3$ con el pórfido $P 2$ sugieren condiciones de cristalización diferentes, indicando variaciones en los procesos formacionales de estos cuerpos.
Según los análisis EDS, es posible que el fluido hidrotermal estuviera cargado con elementos como $\mathrm{Au}, \mathrm{Ag}$ y $\mathrm{Co}$ ya que estos aparecen como trazas asociadas a Py.

La mayor interacción con fluidos hidrotermales tiene lugar en los pórfidos $P 1$ y $P 2$, dando paso al desarrollo de alteraciones Pot, Prop. y QzSer.

El oro parece ser frecuente como inclusiones en sulfuros (principalmente Py) asociados a las zonas de alteración de mayor temperatura (alteración Pot.)

Al igual que las mineralizaciones de Marmato, el control estructural de la mineralización en el SPY tiene una fuerte tendencia W-NW, esto sumado a la similitud en edad (6,3 $\pm 0,7 \mathrm{Ma}$. K/Ar en Hornblenda (Restrepo et al., 1981, en Ramírez et al., 2006) para Marmato y 7+/0,15 Ma. a 6,95+/-0,16 Ma. (Henrichs et al., 2014) para el SPY) y composición de los cuerpos que contienen la mineralización, podrían indicar una correlación genética.

\section{AGRADECIMIENTOS}

A Colombian Mines Corporation quienes facilitaron secciones de los núcleos de perforación sobre los cuales se realizó la presente investigación. Al personal técnico y científico de la Universidad Federal do Rio Grande do Sul, a la CAPES por la financiación en Brasil.

A Isadora Henrichs y Oswaldo Ordoñez por los aportes realizados en investigaciones previas en la zona de estudio. Al Dr. Jhon J. Sánchez por sus observaciones y a los evaluadores cuyas observaciones permitieron mejorar este artículo.

\section{REFERENCIAS}

Alvarán, M. 2006. Consideraciones metalogenéticas del depósito aurífero La Coqueta, distrito minero de Manizales, Villamaría. Tesis de Maestría, Universidad Nacional de Colombia, Bogotá, Colombia. 100p.

Calle, B., y González, H. 1982. Geología y geoquímica de la plancha 186 Riosucio. Informe No. I-1878. INGEOMINAS. Medellín, 120p.

Chamois, P. 2011. Technical report on the Caramanta project, departments of Antioquia and Caldas, Colombia. Scott Wilson Rosceo Postle Associates Inc. Toronto, 72 p.

Colombian Mines Corporation. 2010a. MMI Gold Response Ratio Map. Escala 1:200. Consultado 17 de julio de 2016. http://www.colombianmines.com/ Yarumalito.asp 
Colombian Mines Corporation. 2010b. Yarumalito Simplified Geology Map. Escala 1:200. Colombian Mines Corporation.

González, H., Cossio, U., Maya, M., Vásquez, E., y Holguín, M. 1999. Geología del departamento de Antiquia, Escala 1:400.000, INGEOMINAS.

González, H. 2001. Memoria explicativa mapa geológico departamento de Antioquia, escala 1:400.000, INGEOMINAS.

Guzmán-López, C.A. 2007. Estudio diagenético preliminar de la Formación Amagá. Boletín de Geología, 29(1): 13-20.

Henrichs, I., Frantz. J.C., Marques, J.C., OrdoñezCarmona, O., Castoldi, M., and Sato, K. 2014. Caracterização e geocronologia SHRIMP U-Pb em zircão dar rochas subvulcânicas do sistema pórfiro Yarumalito, Distrito de Marmato, Colômbia. Pesquisas em Geociências, 41(3): 231-242.

López, A., Sierra, G., y Ramírez, D. 2006. Vulcanismo Neógeno en el suroccidente antioqueño y sus implicaciones tectónicas. Boletín Ciencias de la Tierra, 19: $27-42$.

Molina, C. 1990. Principales características geológicas y mineralógicas de la mina el cerro Frontino, Antioquia. Boletín Ciencias de la Tierra, 9: 95-112.

Paris, G., Machette, M., Dart, R.L., and Haller, K.M. 2000. Database and Map of Quaternary faults and folds of Colombia and its offshore regions. Consultado en 24 de mayo 2016. https://pubs.usgs.gov/of/2000/ofr-000284/ofr-00-0284.pdf.

Ramírez, D., López, A., Sierra, G., y Toro, G. 2006. Edad y proveniencia de las rocas volcánico sedimentarias de la Formación Combia en el suroccidente antioqueñoColombia. Boletín de Ciencias de la Tierra, 19: 9-26.

Restrepo, J., Toussaint, J., y González, H. 1981. Edades mio-pliocenas del magmatismo asociado a la Formación Combia, departamentos de Antioquia y Caldas, Colombia. Geología Norandina, 3: 21-26.

Santacruz, L. 2011. Microtermometria de inclusiones fluidas aplicadas al depósito de Marmato. Tesis de pregrado. Universidad Nacional de Colombia, Bogotá, Colombia. 39p.
Sillitoe, R. 2008. Major gold deposits and belts of the North and South American Cordillera: Distribution, tectonomagmatic setting, and metallogenic considerations. Economic Geology, 103: 663-687.

Sillitoe, R. 2010. Porphyry copper systems. Economic Geology, 105: 3-41.

Streckeisen, A. L. 1978. IUGS Subcommission on the Systematics of Igneous Rocks. Classification and Nomenclature of Volcanic Rocks, Lamprophyres, Carbonatites and Melilite Rocks. Recommendations and Suggestions. Neues Jahrbuch für Mineralogie, Abhandlungen, 141: 1-14.

Thompson, R. 2006. Yarumalito Project Technical Report. Thompson consulting. Utah, 105 p.

Van der Hammen, T. 1958. Estratigrafía del Terciario y Maestrichtiano continentales y Tectogénesis de los Andes colombianos (con 7 planchas). Boletín Geológico, 6: 67-128.

Vargas, R. 2005. Evaluación geológica, geoquímica y génesis de la zona de exclusión en Maramato, Caldas, Colombia. Tesis de Maestría. Universidad Nacional de Colombia, Bogotá, Colombia.

Whitney, D., and Evans, B. 2010. Abbreviations for names of rock-forming minerals. American Mineralogist, 95: 185-187.

Trabajo recibido: agosto 21 de 2015

Trabajo aceptado: noviembre 23 de 2016

Manuscrito publicado en internet: diciembre 23 de 2016 